\title{
AN EXPLICIT SHARPNESS INDEX RELATED TO GLOBAL PHASE COHERENCE
}

\author{
Gwendoline Blanchet ${ }^{1}$, Lionel Moisan ${ }^{2}$ \\ ${ }^{1}$ Centre National d'Etudes Spatiales, Toulouse, France \\ ${ }^{2}$ Université Paris Descartes, MAP5, CNRS UMR 8145, Paris, France
}

\begin{abstract}
We propose a definition of a Sharpness Index that is closely related to the notion of Global Phase Coherence recently introduced for automatic image restoration and image quality assessment. Using Gaussian random fields instead of random phase images, we can estimate the probability that a random image has a given Total Variation, which leads us to an explicit formula and a fast algorithm. Theoretical arguments and numerical experiments are given to assess the similarity between the Sharpness Index and the Global Phase Coherence, and an application to non-parametric blind deconvolution is presented, that illustrates the possibilities offered by this new approach.
\end{abstract}

Index Terms - sharpness, global phase coherence, image restoration, blind deconvolution, image quality

\section{INTRODUCTION}

Deriving no-reference image sharpness metrics has several applications in Image Processing, in particular in image restoration (blind and non-blind deconvolution, removal of compression artifacts) and in image quality assessment (typically used to rate compression schemes). It was shown in [1] that a sharpness metric, called Global Phase Coherence (GPC), could be defined by reference to the regularity (Total Variation) of random phase images. This metric was then used in [2] as a part of a more sophisticated sharpness measure, and in [3] to improve the sensitivity of SSIM to blur and aliasing.

In practice, the usefulness of the GPC is limited by the fact that its computation requires several hundreds of MonteCarlo simulations - each one requiring one Discrete Fourier Transform (DFT) - to estimate the first two moments of the distribution of the Total Variation of random phase images. In the present work, we propose a variant of the GPC obtained by considering, instead of random phase images, the equivalent Gaussian random fields, for which we can derive explicit formulas (Section 2). In Section 3, we define the Sharpness Index and compare it to GPC, before we present an application to non-parametric blind deconvolution in Section 4.

This work has been supported by the French National Research Agency under grant ANR-09-BLAN-0029-01.

\section{TOTAL VARIATION OF GAUSSIAN FIELDS}

Let $u: \Omega \rightarrow \mathbb{R}$ be a grey-level $M \times N$ image defined on the rectangular domain $\Omega=\{0, \ldots, M-1\} \times\{0, \ldots, N-1\}$. We call standard white noise a random image $W: \Omega \rightarrow \mathbb{R}$ such that all $W(\mathbf{x})$ are independent random variables distributed according to a the Gaussian distribution $\mathcal{N}\left(0,|\Omega|^{-1}\right)$ (that is, mean 0 and variance $\left.(M N)^{-1}\right)$. Note that this normalization ensures that $\mathbb{E}\|W\|^{2}=1$, where $\mathbb{E}$ denotes the expectation and $\|W\|^{2}=\sum_{\mathbf{x} \in \Omega} W(\mathbf{x})^{2}$. Combining $u$ and $W$ with a periodic convolution, we obtain a new random image $U=u \star W$ defined on $\Omega$ by

$$
U(\mathbf{x})=\sum_{\mathbf{y} \in \Omega} W(\mathbf{y}) \dot{u}(\mathbf{x}-\mathbf{y})
$$

where $\dot{u}$ means the $\Omega$-periodization of $u$ (that is, $\dot{u}$ is $N$ periodic along each coordinate). It is easy to prove that this definition ensures that $U$ is a stationnary Gaussian random field with mean $0_{\Omega}$ and covariance matrix

$$
\mathbb{E} U(\mathbf{x}) U\left(\mathbf{x}^{\prime}\right)=\frac{1}{|\Omega|} \sum_{\mathbf{y} \in \Omega} u(\mathbf{y}) \dot{u}\left(\mathbf{y}+\mathbf{x}^{\prime}-\mathbf{x}\right) .
$$

We now consider the notion of (periodic) Total Variation, that associates to an image $v: \Omega \rightarrow \mathbb{R}$ the value

$$
\operatorname{TV}(v):=\left\|\partial_{x} v\right\|_{1}+\left\|\partial_{y} v\right\|_{1}=\sum_{\mathbf{x} \in \Omega}\left|\partial_{x} v(\mathbf{x})\right|+\left|\partial_{y} v(\mathbf{x})\right|,
$$

where $\partial_{x} v(x, y)=\dot{v}(x+1, y)-v(x, y)$ and $\partial_{y} v(x, y)=$ $\dot{v}(x, y+1)-v(x, y)$. This is a regularity measure that has been extensively used in image processing since the seminal paper of Rudin, Osher and Fatemi [4]. In order to establish the main theoretical result of this paper (Theorem 1 below), we need to introduce the gradient cross-correlation function defined by

$$
\forall \mathbf{z} \in \Omega, \quad \Gamma(\mathbf{z})=\sum_{\mathbf{y} \in \Omega} \nabla \dot{u}(\mathbf{y}) \cdot \nabla \dot{u}(\mathbf{y}+\mathbf{z})^{T},
$$

where $\nabla \dot{u}=\left(\partial_{x} \dot{u}, \partial_{y} \dot{u}\right)^{T}$ is the (periodic) gradient of $u$. Note that $\Gamma(\mathbf{z})$ is a $2 \times 2$ matrix, that can be written under the form

$$
\Gamma(\mathbf{z})=\left(\begin{array}{cc}
\Gamma_{x x}(\mathbf{z}) & \Gamma_{x y}(\mathbf{z}) \\
\Gamma_{y x}(\mathbf{z}) & \Gamma_{y y}(\mathbf{z})
\end{array}\right) .
$$

Now we can state 
Theorem 1 The expectation and the variance of $\mathrm{TV}(U)$ are

$$
\begin{gathered}
\mathbb{E} \operatorname{TV}(U)=\left(\alpha_{x}+\alpha_{y}\right) \sqrt{\frac{2|\Omega|}{\pi}}, \\
\operatorname{Var} \operatorname{TV}(U)=\frac{2}{\pi} \sum_{\mathbf{z} \in \Omega}\left[\alpha_{x}^{2} \cdot \omega\left(\frac{\Gamma_{x x}(\mathbf{z})}{\alpha_{x}^{2}}\right)+\right. \\
\left.2 \alpha_{x} \alpha_{y} \cdot \omega\left(\frac{\Gamma_{x y}(\mathbf{z})}{\alpha_{x} \alpha_{y}}\right)+\alpha_{y}^{2} \cdot \omega\left(\frac{\Gamma_{y y}(\mathbf{z})}{\alpha_{y}^{2}}\right)\right],
\end{gathered}
$$

where $\alpha_{x}=\left\|\partial_{x} u\right\|_{2}, \alpha_{y}=\left\|\partial_{y} u\right\|_{2}, \Gamma$ is the cross-correlation matrix of $\nabla u$ and $\omega$ is the function defined by

$$
\forall t \in[-1,1], \quad \omega(t)=t \arcsin t+\sqrt{1-t^{2}}-1 .
$$

Proof: We have by linearity $\partial_{x} U=\left(\partial_{x} u\right) \star W$, so that the discrete random field $\partial_{x} U$ is a stationnary Gaussian field whose marginal distributions have zero mean and variance

$$
\mathbb{E}\left(\partial_{x} U(\mathbf{x})\right)^{2}=\sum_{\mathbf{y} \in \Omega} \frac{1}{|\Omega|}\left(\partial_{x} \dot{u}(\mathbf{x}-\mathbf{y})\right)^{2}=\frac{\alpha_{x}^{2}}{|\Omega|},
$$

and then a standard computation leads to

$$
\forall \mathbf{x} \in \Omega, \quad \mathbb{E}\left|\partial_{x} U(\mathbf{x})\right|=\sqrt{\frac{2 \alpha_{x}^{2}}{\pi|\Omega|}} .
$$

Using a similar reasonning on $\partial_{y} U$, we obtain that the expectation of $\operatorname{TV}(U)=\sum_{\mathbf{x} \in \Omega}\left|\partial_{x} U(\mathbf{x})\right|+\left|\partial_{y} U(\mathbf{x})\right|$ is given by (2) as announced.

We now consider the variance of $\operatorname{TV}(U)$. We have

$$
\mathbb{E} \operatorname{TV}(U)^{2}=\sum_{a, b \in\{x, y\}} \sum_{\mathbf{x}, \mathbf{y} \in \Omega} \mathbb{E}\left|\partial_{a} U(\mathbf{x}) \partial_{b} U(\mathbf{y})\right|,
$$

and the stationnarity of $\nabla U$ entails (with $\mathbf{z}=\mathbf{y}-\mathbf{x}$ )

$$
\mathbb{E} \operatorname{TV}(U)^{2}=|\Omega| \sum_{a, b \in\{x, y\}} \sum_{\mathbf{z} \in \Omega} \mathbb{E}\left|\partial_{a} U(0) \partial_{b} U(\mathbf{z})\right| .
$$

Each term of this double sum can be written under the form $\mathbb{E}|X Y|$, where $(X, Y)$ is a zero-mean 2-dimensional Gaussian vector with covariance matrix

$$
\left(\begin{array}{cc}
\mathbb{E} X^{2} & \mathbb{E} X Y \\
\mathbb{E} X Y & \mathbb{E} Y^{2}
\end{array}\right)
$$

For the term of (5) corresponding to $a=x$ and $b=y$ for example, we have $X=\partial_{x} U(0)$ and $Y=\partial_{y} U(\mathbf{z})$, thus

$$
\begin{aligned}
\mathbb{E} X Y & =\mathbb{E} \sum_{\mathbf{x}, \mathbf{y} \in \Omega} \partial_{x} \dot{u}(-\mathbf{x}) \partial_{y} \dot{u}(\mathbf{z}-\mathbf{y}) W(\mathbf{x}) W(\mathbf{y}) \\
& =\frac{1}{|\Omega|} \sum_{\mathbf{x} \in \Omega} \partial_{x} \dot{u}(\mathbf{x}) \partial_{y} \dot{u}(\mathbf{z}+\mathbf{x}) \\
& =\frac{1}{|\Omega|} \Gamma_{x y}(\mathbf{z})
\end{aligned}
$$

and the covariance matrix of $(X, Y)$ is

$$
\frac{1}{|\Omega|}\left(\begin{array}{cc}
\alpha_{x}^{2} & \Gamma_{x y}(\mathbf{z}) \\
\Gamma_{x y}(\mathbf{z}) & \alpha_{y}^{2}
\end{array}\right),
$$

so that by Lemma 1 below we obtain that

$$
\mathbb{E}|X Y|=\frac{2 \alpha_{x} \alpha_{y}}{\pi|\Omega|} \cdot \tilde{\omega}\left(\frac{\Gamma_{x y}(\mathbf{z})}{\alpha_{x} \alpha_{y}}\right),
$$

with $\tilde{\omega}(t)=\cos (\arcsin t)+\arcsin t \cdot \sin (\arcsin t)=$ $\sqrt{1-t^{2}}+t \arcsin t=\omega(t)+1$. Combining all terms arising from (5), we finally obtain that

$$
\mathbb{E} \operatorname{TV}(U)^{2}=\frac{2}{\pi} \sum_{a, b \in\{x, y\}} \sum_{\mathbf{z} \in \Omega} \alpha_{a} \alpha_{b} \cdot \tilde{\omega}\left(\frac{\Gamma_{a b}(\mathbf{z})}{\alpha_{a} \alpha_{b}}\right),
$$

where the contributions of $(a, b)=(x, y)$ and $(a, b)=(y, x)$ are equal since $\Gamma_{y x}(\mathbf{z})=\Gamma_{y x}(-\mathbf{z})$. In the end, the announced result arises from $\operatorname{Var} \operatorname{TV}(U)=\mathbb{E} \operatorname{TV}(U)^{2}-(\mathbb{E} \operatorname{TV}(U))^{2}$, which simply amounts to change $\tilde{\omega}$ into $\omega$ in (6).

Lemma 1 let $Z=(X, Y)^{T}$ be a Gaussian random vector with zero mean and covariance matrix

$$
\mathbb{E}\left(Z Z^{T}\right)=\left(\begin{array}{cc}
p^{2} & p q \sin \theta \\
p q \sin \theta & q^{2}
\end{array}\right) .
$$

Then, one has $\mathbb{E}|X Y|=\frac{2|p q|}{\pi}(\cos \theta+\theta \sin \theta)$.

The proof results from standard integration calculus.

\section{A NEW SHARPNESS INDEX}

\subsection{Definition and relation to GPC}

Using Theorem 1, we are now in position of defining a new Sharpness Index, inspired from the GPC developped in [1].

Definition 1 The Sharpness Index of an image $u: \Omega \rightarrow \mathbb{R}$ is

$$
S I(u)=-\log _{10} \Phi\left(\frac{\mu-\mathrm{TV}(u)}{\sigma}\right),
$$

where $\mu=\mathbb{E} \operatorname{TV}(U)$ and $\sigma^{2}=\operatorname{Var} \operatorname{TV}(U)$ are defined in Theorem 1, and $\Phi(x)=(2 \pi)^{-1 / 2} \int_{x}^{+\infty} e^{-t^{2} / 2} d t$ is the tail of the Gauss distribution.

As in [1], we replace the uncomputable probability $\mathbb{P}\{T V(U) \leq T V(u)\}$ by its Gaussian approximation based on the first two moments of $T V(U)$ (which is reasonable because $T V(U)$ is very near to be Gaussian in practice). Thus, the fundamental difference between $G P C(u)$ and $S I(u)$ relies in the random field $U$ that is used: random phase images for $\operatorname{GPC}(u)$, a Gaussian random field for $S I(u)$. As we shall see further, these two random fields lead to very similar properties, but the great advantage of the Gaussian random field is that it provides explicit formulas for $S I(u)$, whereas the estimation of $G P C(u)$ requires heavy Monte-Carlo simulations. In that sense, one can see the Sharpness Index we just defined as an explicit ersatz of the GPC. 


\subsection{Numerical computation}

As for the GPC, we have to consider two potential issues in the estimation of the Sharpness Index: the fact that we assume periodic images (because we use a periodic TV and periodic convolutions), and the fact that actual images are quantized (on 256 grey levels, or more). To avoid undesirable effects, we thus apply the pre-processing mentioned in [1] before we compute the Sharpness Index. This leads us to the more robust Sharpness Index

$$
S I^{\prime}(v)=S I(Q(\operatorname{per}(v))),
$$

where $Q$ is a dequantization operator (a $(1 / 2,1 / 2)$ Fourierbased translation), and $\operatorname{per}(v)$ the periodic component of $v$ (avoiding periodization artefacts) defined in [5]. Note that the computation of the Fourier Transform of $u=Q(\operatorname{per}(v))$ only requires two DFTs. As concerns the computation of $S I(u)$, it involves the computation of the gradient $\nabla u=$ $\left(\partial_{x} u, \partial_{y} u\right)^{T}$, the norms $\alpha_{x}=\left\|\partial_{x} u\right\|_{2}$ and $\alpha_{y}=\left\|\partial_{y} u\right\|_{2}$, and the cross-correlation images $\Gamma_{x x}, \Gamma_{x y}, \Gamma_{y y}$, which can be quickly computed in Fourier Domain thanks to the relation $\widehat{\Gamma_{a b}}=\widehat{\partial_{a} u} * \widehat{\partial_{b} u}$. Overall, the computation of $S I^{\prime}(v)$ requires the computation of 6 DFTs ( 2 to compute $\hat{u}$ from $v, 1$ to get $u$, and 3 to get the cross-correlation images). In practice, the computation of $S I^{\prime}$ on a $512 \times 512$ image takes about $200 \mathrm{~ms}$ on a recent laptop with a $\mathrm{C}$ implementation (as a comparison, computing the GPC with $N=1000$ Monte-Carlo samples takes about 1 minute).

\subsection{Comparison between Sharpness Index and GPC}

The aim of the present paper is to show that GPC can be advantageously replaced by the Sharpness Index without loosing its properties. From a theoretical viewpoint, it is interesting to notice that random phase images (obtained by phase randomization in Fourier Domain) and the equivalent Gaussian images (obtained by convolution with a white noise) produce very similar images visually. In fact, they only differ in Fourier Domain by a mutilplicative Rayleigh noise [6]. Hence, one can expect that the Sharpness Index and the GPC will essentially measure the same thing. Indeed, $S I(u)$ and $G P C(u)$ are the same combination of three numbers: $T V(u), \mu=\mathbb{E} T V(U)$, and $\sigma^{2}=\operatorname{Var} T V(U), U$ being a different random field in each case. Experimentally, we observed that the values of $\mu$ obtained for $G P C(u)$ and $S I(u)$ (that is, the average total variation of a random phase image and the equivalent Gaussian image) were very close, with typical relative differences below $1 \%$. As concerns $\sigma$, however, there is a real difference between the two random fields, the value being systematically higher for Gaussian fields (the ratio seems to be around 7-8 in general). Hence, we expect $S I(u)$ to be significantly lower than $G P C(u)$, but as we shall see now it does not have important consequences in practice, since both quantities behave the same with respect to image changes.
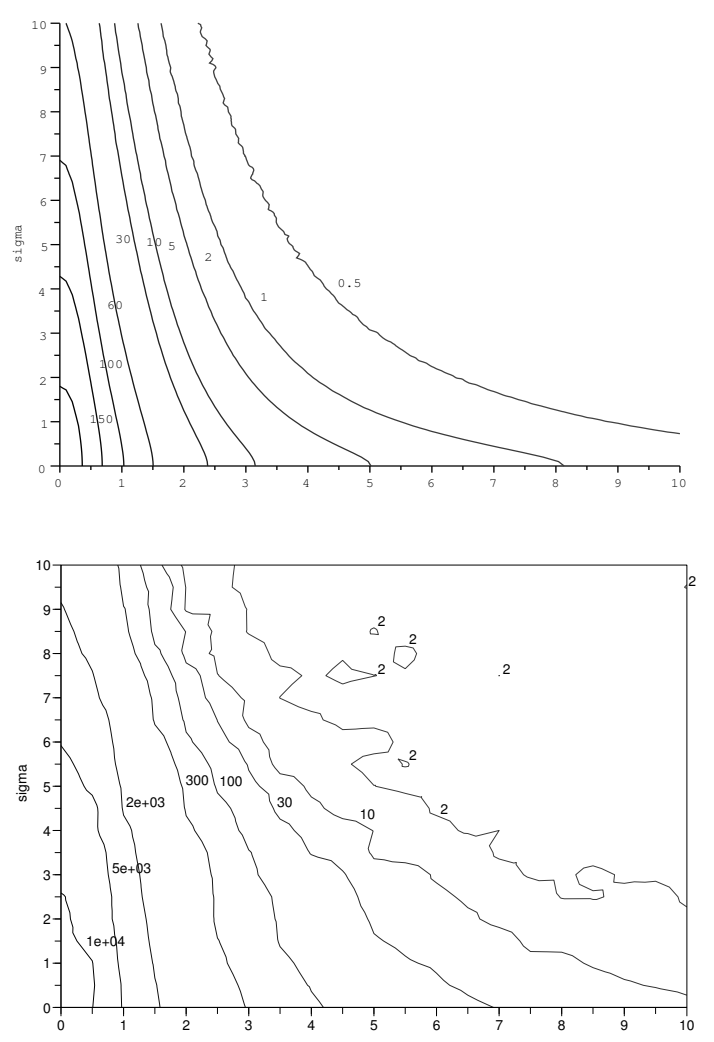

Fig. 1. 2D blur-noise diagrams of Lacornou image, corresponding to the Sharpness Index (top) and the GPC (bottom).

To compare the behavior of $S I(u)$ and $G P C(u)$ as functions of $u$, we compute as in [1] the 2D blur-noise diagram, that is, the function

$$
f(r, \sigma)=S I^{\prime}\left(g_{r} \star u+\sigma N\right),
$$

where $g_{r}$ is the 2-D Gaussian convolution kernel with standard deviation $r$, and $N$ a white noise image with unit variance in each pixel. This blur-noise diagram is compared to the one obtained for GPC in [1] on the same image (Lacornou), and the results are shown on Fig.1. As we can see, the absolutes values of $S I(u)$ and $G P C(u)$ differ, but their relative behavior with respect to noise and blur is quite similar.

\section{APPLICATION TO BLIND DECONVOLUTION}

In [1], an application of the GPC to parametric blind deconvolution was presented. It was shown that an appropriate value of the standard deviation $r$ of a Gaussian kernel could be selected in a Wiener blind deconvolution scheme by maximizing the GPC of the deconvolved image. We applied the same protocol by replacing the GPC with the Sharpness Index, and we found identical results, that is, the same value of $r$. Rather than reproducing the identical curve we obtained (up to the scale change we discussed above), we propose here to illus- 
trate the interest of the Sharpness Index by considering a more challenging problem: non-parametric blind deconvolution.

Instead of formulating blind deconvolution as an inverse problem associated to an unknwown convolution kernel, we propose to use a more direct approach: given an image $u$, find the symmetric convolution kernel $k$ such that $S I(k \star u)$ is maximal. Solving this optimization problem is not easy, because the function $F: k \mapsto S I(k \star u)$ is neither concave nor smooth (due to the presence of the TV operator). This is why we adopt a very simple (though probably not optimal in terms of computation time) strategy, called stochastic minimization:

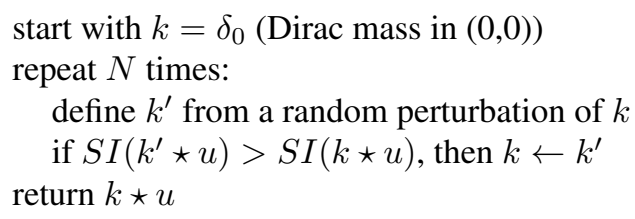

In order to limit the dimensionality of the problem, we considered separable kernels $k$ with a small $(21 \times 21)$ support. Thus, considering the symmetry constraint on $k$, the exploration space has dimension 10 (we chose to maintain $k(0)=$ 1 , which is equivalent to the normalization $\sum_{\mathbf{x}} k(\mathbf{x})=1$ since $S I(\lambda u)=S I(u)$ for any $\lambda \neq 0)$. The algorithm above could be trapped in a local maximum of the map $F$, but we did not observe this in practice. It might be that the map $F$ associated to typical images only has one local maximum (which is global), but at this time we have no evidence of this. In the experiments we performed on several classical images, we used $N=10,000$, which seemed sufficient since we observed little improvement on the Sharpness Index after $N=1000$. Also, running the algorithm several times always led to the same solution (up to a good level of precision).

The deconvolution algorithm we just presented was applied to several classical images, without applying any prior blur or noise. The results were surprisingly good, considering that no regularity constraint was imposed on the blur kernel or on the deconvolved image, as is generally done in classical blind deconvolution algorithms. Notice, however, that for some images the result was a bit too much deconvolved (in the sense that a careful examination of the result revealed the presence of ringing around edges). A representative example is shown on Fig. 2.

\section{CONCLUSION}

While the Global Phase Coherence defined in [1] requires heavy Monte-Carlo simulations to be estimated, the Sharpness Index we proposed here is computed exactly with only 6 Discrete Fourier Transforms. Though, these two image quality indices exhibit very similar behaviors, in particular a sensitivity to image sharpness in a strong sense, that is, by opposition to several causes of the loss of details in an image (not only blur, but also noise, aliasing, ringing). Hence, considering the Sharpness Index instead of GPC opens interesting

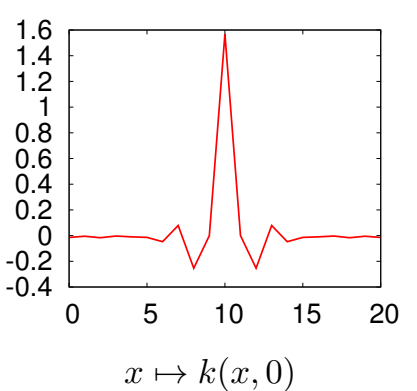

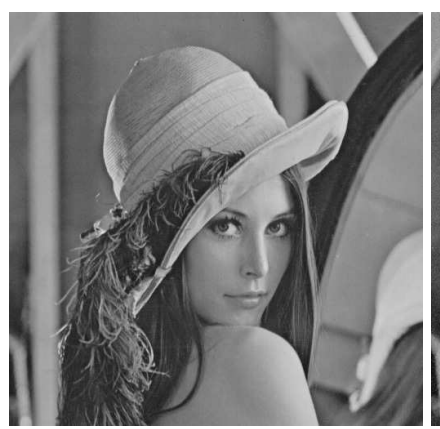

$u$

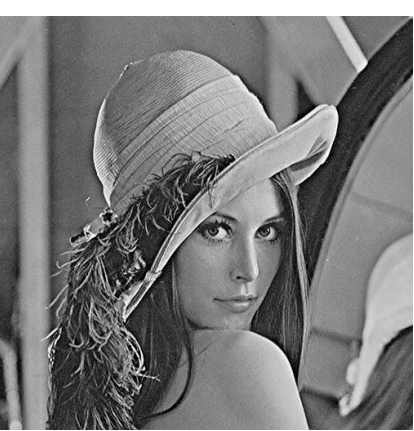

$k \star u$
Fig. 2. Blind deconvolution of Lena image. Selecting the separable symmetric $21 \times 21$ kernel $k$ that maximizes the Sharpness Index of $k \star u$ ( $u$ being the original Lena image, bottom left) results in a sharper image (bottom right) that reveal some details of the original image, while keeping noise and ringing at an acceptable level.

perspectives, both from a theoretical viewpoint (the explicit formulas will probably ease further analyses) and as concerns applications (in particular image restoration), for which computation time and estimation errors are no more a limitation.

\section{REFERENCES}

[1] G. Blanchet, L. Moisan, and B. Rougé, "Measuring the global phase coherence of an image," in Proc. Int. Conf. on Image Processing, 2008, pp. 1176-1179.

[2] C. Vu and D. Chandler, "S3: A spectral and spatial sharpness measure," in MMEDIA'09, 2009, pp. 37-43.

[3] D. Wang, W. Ding, Y. Man, and L. Cui, “A joint image quality assessment method based on global phase coherence and structural similarity," Int. Cong. on Image and Signal Processing (CISP), vol. 5, pp. 2307-2311, 2010.

[4] L. Rudin, S. Osher, and C. Fatemi, "Nonlinear total variation based noise removal algorithm," Physica D, vol. 60, pp. 259-268, 1992.

[5] L. Moisan, "Periodic plus smooth image decomposition," J. Math. Imaging Vision, vol. 39:2, pp. 161-179, 2011.

[6] B. Galerne, Y. Gousseau, and J.-M. Morel, "Random phase textures: Theory and synthesis," IEEE Trans. on Image Processing, vol. 20:1, pp. 257-267, 2011. 